(2) Open Access Full Text Article

\title{
Improving patient-physician dialog: commentary on the results of the MS Choices survey
}

\author{
This article was published in the following Dove Press journal: \\ Patient Preference and Adherence \\ 16 February 2012 \\ Number of times this article has been viewed
}

\author{
Alessandra Lugaresi ${ }^{1}$ \\ Tjalf Ziemssen ${ }^{2}$ \\ Celia Oreja-Guevara ${ }^{3}$ \\ Delyth Thomas ${ }^{4}$ \\ Elisabetta Verdun ${ }^{5}$ \\ 'Department of Neuroscience and \\ Imaging, University "G d'Annunzio", \\ Chieti, Italy; ${ }^{2}$ Center of Clinical \\ Neuroscience, Neurological \\ University Clinic, Dresden University \\ of Technology, Dresden, Germany; \\ ${ }^{3}$ Multiple Sclerosis Unit, Department \\ of Neurology, Health Research \\ Institute (IdiPAZ), University Hospital \\ La Paz, Madrid, Spain; ${ }^{4}$ Wye Valley \\ NHS Trust, Hereford, United Kingdom; \\ ${ }^{5}$ Global Medical Affairs Neurology, \\ Merck Serono S.A. - Geneva, \\ Switzerland
}

Correspondence: Alessandra Lugaresi Department of Neuroscience and Imaging, University “G d'Annunzio”, c/o Centro Sclerosi Multipla, Ospedale Clinicizzato "SS Annunziata", Via dei Vestini 5, 66100 Chieti, Italy Tel +39 087I 358532

Email a.lugaresi@unich.it

\begin{abstract}
Achieving good long- and short-term adherence to treatment for chronic diseases is important if patients are to gain the full benefits of treatment. Several barriers to adherence in multiple sclerosis (MS) have been identified and the healthcare team should work with patients to help them to overcome these obstacles. The MS Choices survey explored patient and physician perspectives on key aspects of MS diagnosis, treatment adherence, and disease management with the aim of improving understanding of the factors that influence patient behavior regarding treatment adherence. The survey found some important differences between patient and physician responses and here these findings are discussed in the context of personal clinical experience. Further, the possible implications of these findings for routine practice have been considered, and strategies that should be employed by MS physicians and nurses to help patients to adhere to their prescribed treatment are suggested.
\end{abstract}

Keywords: multiple sclerosis, treatment adherence, treatment initiation, patient-physician dialog, psychological profiling

\section{Introduction}

Among patients with chronic diseases requiring long-term medication, achieving and maintaining good adherence to treatment is both critical and problematic. ${ }^{1-3}$ Many factors can contribute to poor adherence over the long-term, including treatment fatigue, forgetfulness, and treatment-related side effects. ${ }^{2,4-6}$ In patients with multiple sclerosis (MS), additional disease-related factors may also contribute to poor adherence. In a disease characterized by relapses and periods of remission, patients may think that they do not need to take their treatment when they feel well, or they may perceive that their treatment is not working in the event of a relapse or simply because symptoms persist despite treatment. Perceived lack of efficacy was found to be the main cause of treatment discontinuation in a cohort of Italian patients. ${ }^{?}$

Clinical experience tells us that specific symptoms of MS can also impact on adherence; for example, impaired visual function or manual dexterity, or spasticity, may affect the patient's ability to perform injections. Cognitive impairment and depression may also make it difficult for the patient to fully understand the importance of maintaining a regular treatment pattern, whereas anxiety or loss of memory may make it difficult for patients to remember when to take their medication, or may lead to confusion over when the last dose was taken. Moreover, with all established firstline treatments still requiring regular parenteral administration, needle phobia, fear of injection, and concerns over the ability to self-inject correctly present significant barriers to adherence for many patients with $\mathrm{MS}^{8}$ 
The importance of achieving good treatment adherence is clear: poor adherence or breaks in treatment can limit treatment benefits and have a detrimental effect on disease-related outcomes, such as axonal damage. ${ }^{910}$ It has been suggested that addressing poor adherence has the potential to have a greater impact on treatment efficacy than the development of new drugs, ${ }^{11}$ and could also achieve a better cost:benefit ratio. From an economic perspective, early and consistent treatment should also provide benefits by reducing healthcare costs associated with relapses or increased disability. ${ }^{9}$

It is imperative that adherence is addressed both immediately after treatment initiation and over the longer-term. The World Health Organization's definition of adherence ${ }^{1}$ highlights the role of the patient as the key decision maker in the treatment process and the driver of treatment success. Therefore, the authors believe that it is important to consider which factors will motivate patients at different stages of their disease. That the motivating factors may differ between patients and change over time should also be recognized. Patients who are "doing well" or have just started treatment must receive the appropriate support to minimize the likelihood that they will take breaks from or permanently discontinue treatment. In addition, the authors have found in their clinics that different patients will be more concerned over different aspects of treatment, such as efficacy, side effects, or convenience. For example, in the authors' experience, side effects, in particular injection-site reactions, are of great concern for some patients such as young women who may find their appearance embarrassing. Throughout their treatment, patients need to be helped to understand that they may have to make some sacrifices now (eg, having to accept "flu-like" symptoms, which are common early in treatment but tend to abate over time) to achieve better long-term outcomes. Through direct contact with patients, the healthcare team has the potential to impact patient adherence positively. ${ }^{5}$ However, for this to be possible, the authors believe that the healthcare team needs accurate information on patients' perceptions of their disease and treatment. A good patientphysician or patient-nurse relationship, in which patients feel comfortable voicing their honest opinions, and a good understanding of patients' needs, wants, and expectations of treatment, are also essential.

Several approaches, including the provision of injection devices and patient support programs, have been employed with the aim of improving the patient's experience of treatment, and thus adherence. Of these, injection devices have the potential to help patients to overcome some of the psychological barriers to self-injection, for example by keeping the needle hidden throughout the injection process. ${ }^{12}$ Whether injection devices increase adherence has yet to be confirmed, but a recent observational study of 1077 patients with MS in 15 countries found that use of an injection device was the strongest predictor of treatment adherence over 24 months. ${ }^{13}$ Reductions in the occurrence of injection-site reactions and injection-related discomfort with injection device use have also been reported. ${ }^{2,14,15}$

The MS Choices survey was carried out to investigate patient and physician perspectives on key aspects of MS diagnosis, treatment adherence, and disease management. It was hoped that the survey results would improve understanding of the extent of poor adherence and cultural/economic influences on treatment initiation and adherence. Results of the survey have been published ${ }^{16}$ a commentary on this paper is provided here, discussing the findings in the context of the authors' personal clinical experience, and suggesting the implications of these findings for routine practice.

\section{The MS Choices survey Background of the survey}

The MS Choices survey was conducted in seven countries (Australia, Canada, France, Germany, Italy, Spain, and the UK) across three continents. Patients who had been diagnosed with MS for $\geq 1$ year and were currently receiving disease-modifying drug (DMD) treatment and neurologists with 3-30 years of clinical experience treating on average $\geq 15$ patients with MS per month were invited to participate. Overall, 331 patients and 280 neurologists took part. Participation required that two questionnaires (one each for patients and physicians) that had previously undergone pilot testing were completed; both contained closed- and open-answer questions. Patients completed a semi-structured paper questionnaire comprising 37 questions (including two free-text questions) that took approximately 20 minutes to complete. Physicians completed an online questionnaire comprising 29 questions (24 closed answers and five percentage estimations) that took approximately 15 minutes to complete. The questionnaires can be found in the published results of the MS Choices survey. ${ }^{16}$

\section{Summary of results}

Several differences were found between patient and physician responses, particularly for questions relating to treatment adherence. Almost twice as many patients reported taking a treatment break as the number estimated by neurologists. Differences were also seen between the reasons for poor adherence given by patients and physicians. Although both groups reported side effects as the main reason for poor 
adherence, this response was given by almost twice as many physicians as patients ( $82 \%$ vs $42 \%$ ). Whereas $54 \%$ of physicians thought patients would stop treatment because they thought it was not working, only $9 \%$ of patients cited this reason. In addition, $13 \%$ of patients reported that they would stop treatment as they felt drained or fed up with treatment. It is important to consider that a patient's reasons for feeling fed up could include the perception that they were not gaining benefit from their treatment.

Differences were also seen between physician and patient responses regarding the timing of treatment initiation and patient involvement in treatment decisions. Notably, whereas $47 \%$ of physicians thought that patients were fully involved in deciding their treatment, only $23 \%$ of patients felt that they had discussed treatment with their healthcare team and had been responsible for their treatment selection. Regional differences in responses regarding these practices were also observed.

The authors of the survey concluded that neurologists may underestimate the scale of poor adherence to DMDs. This conclusion is understandable as $59 \%$ of physicians did not consider adherence to be a problem, yet a study of 2648 patients with MS, in which adherence was defined as not missing any DMD injection in the 4 weeks before the study, reported that one-quarter of patients were not adherent to therapy. ${ }^{17,18}$ As the MS Choices authors noted, assuming good adherence could result in a treatment being incorrectly considered as ineffective. The concern in such a situation is that this may result in unnecessary treatment escalation, exposing the patient to the potential risks associated with more aggressive treatment. It was also concluded that disparities exist between patients' and physicians' opinions regarding the reasons for poor adherence, and the authors proposed that improving the patient-physician dialog may increase adherence to DMDs.

\section{Commentary on the MS Choices survey findings \\ Patients and physicians completing the survey}

The survey was designed to involve representative patient and physician populations in countries with a high prevalence of MS and good access to treatment. Both patient and physician numbers were distributed evenly across participating countries. Neurologists were identified by a specialist provider and were screened using the eligibility questions before completing the questionnaire to ensure that they had substantial and ongoing experience of treating patients with MS.
All patients had been diagnosed with $\mathrm{MS}$ for $\geq 1$ year and were currently taking DMD treatment, ensuring that responses related to current practices. As newly diagnosed patients were excluded, experiences of those who started treatment immediately after diagnosis were not captured.

Although the above requirements ensured that a population representative of patients with MS with experience of DMD treatment was included in the survey, there were some limitations. As there was no upper limit for duration of MS, many responses (particularly those relating to treatment initiation) were reliant on patients' memory. Considering this, it would have been interesting to see how responses differed between groups of patients stratified by, for example, time from diagnosis or duration of therapy. It would also have been interesting to include MS nurses in the survey to see how their perceptions of patient experience of treatment differed from those of physicians and patients themselves.

\section{Discrepancies between patient}

\section{and physician responses}

For all topics explored, discrepancies were found between patient and physician responses, as discussed in more detail for specific topics below. There are several possible reasons, in general, for such discrepancies. Physicians and patients were selected independently and not "matched;" therefore, these could reflect true differences in treatment practices. Questions in the patient and physician questionnaires were phrased differently and may have explored different specific aspects of the treatment experience. Furthermore, physicians probably responded in general terms about their behavior at the time of the survey, whereas patients would have reported their own personal experience relating to practice at the time of their diagnosis, and may depend on imperfect recall; therefore, general practices were most likely compared with individual cases. For both questionnaires, many questions included "fixed" answers, which may have prompted a particular response. A previous study also revealed differences in opinion between patients with MS and physicians when asked about treatment continuation and the risks associated with natalizumab treatment. ${ }^{19}$

\section{Regional differences in responses}

Several differences between countries were identified in the MS Choices survey. While the reasons for cultural/regional differences were not explored, these could reflect different processes within the healthcare system that influence, for example, the time at which DMD treatment can occur, different access to healthcare or differences in healthcare 
funding, and cultural differences such as the desire of patients to be involved in decisions regarding their treatment. It is important to remember that regional differences can also exist within a single country. For example, in some areas of Spain, a central committee must approve initiation of DMD therapy, incurring delays of 3-6 months until a treatment can be started, whereas other regions have no committee, so immediate initiation of therapy is possible. Thus, the reasons for the observed differences are likely complex.

\section{Lessons from MS Choices Treatment initiation}

\section{Timing of treatment initiation}

An interesting finding was the large discrepancy between the timing of treatment initiation after diagnosis reported by patients and physicians. In general, most physicians reported that they treated patients during the first 6 months following diagnosis, whereas over a third of patients reported waiting more than a year after diagnosis before starting treatment. Unfortunately, the duration of treatment was not captured in the survey, so it is not known whether this difference reflected, for example, changing practices over time or real differences owing to patients and physicians not having been "matched."

The time from diagnosis to the start of treatment also differed between countries. Most ( $\geq 80 \%$ ) physicians in Germany and Australia reported starting treatment within 2 months of diagnosis, while a minority $(<40 \%)$ from the UK and Spain did so. In Italy, Canada, and France, approximately half of physicians reported starting treatment within 2 months of diagnosis. This finding may relate to differences in the healthcare systems, as noted above.

\section{Delaying the start of treatment}

Reasons for delaying treatment initiation were not captured in the survey, but should be considered. The physician may deliberately delay treatment for some patients, for example, to allow time for the patient to accept the MS diagnosis and the need for treatment. As a patient's emotional state can influence their ability to accept their situation, the same management schedule cannot be applied to all patients. Where there is a delay in starting treatment, it is important that the reasons for this decision are explained to the patient, as improving patient engagement could have a positive effect on adherence. Furthermore, a delay in starting treatment could suggest that treatment is not very important, which could influence a patient's subsequent attitude to treatment and reduce adherence. On the contrary, a short delay might be necessary to allow patients to adjust to their diagnosis, overcome depression and anxiety, and understand not only the importance of treatment but also the possible side effects. Considering that patients with MS can experience psychological distress, even in the early stages of their disease, ${ }^{20}$ allowing patients time to come to terms with their situation could help to avoid discontinuations in the first 6 months of treatment. Preventing early discontinuations may be particularly important to achieve good long-term adherence and outcomes. A retrospective review of Canadian patients with MS receiving interferon- $\beta$ therapy found that the highest proportion of treatment interruptions occurred in the first 6 months after starting therapy. ${ }^{4}$ Crucially, the level of information given to a patient at diagnosis regarding treatment and the need for treatment should be tailored to the individual as far as possible.

\section{Treatment adherence}

Physicians' and patients' perspectives on reasons for poor treatment adherence

Side effects were the main reason given by both patients $(42 \%)$ and physicians $(82 \%)$ for poor treatment adherence, although clearly physicians considered side effects a more important driver of nonadherence than did patients. Other differences between patient and physician responses to questions exploring reasons for poor treatment adherence were also seen. From the physician's perspective, the vast majority of patients stop treatment for reasons associated with efficacy or side effects. In contrast, many patients cited being emotionally drained or fed up with treatment, practical issues relating to injecting, or forgetfulness. None of these reasons were cited by physicians, although physicians might have considered these psychological issues to be contributors to "perceived lack of efficacy," and might have considered reasons for nonadherence as a balance between the pros and cons of treatment and overall impact on patient quality of life.

For many patients, fitting the need to inject around their lifestyle was the biggest challenge, and how to help patients to overcome this issue should be addressed by their medical team. The responses regarding reasons for poor adherence suggest that physicians think mostly in terms of the effect of treatment on physical symptoms, and not in terms of the overall effect that treatment may have on the patient's lifestyle. This finding highlights the need to educate physicians about the issues that are important to patients, the importance of flexibility in treatment administration, and the need to have more discussions with their patients, ideally asking open questions about any concerns the patient may have. 
In the authors' experience, patient expectation of therapy is possibly the most important factor predicting adherence: those with unrealistic expectations are likely to discontinue treatment early, often at the time of first relapse. Periodic checks of patient expectations can be conducted using a simple questionnaire that the patient completes while waiting to see the neurologist or nurse. Gaining the MS nurse's perspective on the issues that are important to patients would have been interesting, since nurses may have been more aware than physicians of problems such as being emotionally drained by the need for regular injections.

\section{Are scheduled treatment breaks justified?}

Differences were also seen in the proportion of patients who reported taking a treatment break (31\%), which was higher than the proportion of patients estimated by physicians as having taken a treatment break $(17 \%)$. This may have reflected the different wording of the question in the two questionnaires, since "treatment break" was defined in the question for patients but not for physicians. The definition of treatment break given to patients (not taking treatment for " 1 day or longer") is more stringent than that applied in many studies, which may be closer to the physicians' understanding of a "treatment break." The perception of adherence may also differ between physicians, with younger physicians more open to the issue of poor treatment adherence.

Although $>90 \%$ of physicians reported having at least one patient who had taken a break from or stopped their MS therapy, 59\% did not think compliance was an issue. One explanation could be that physicians authorize treatment breaks and consider patients taking such a break to be compliant. Based on the authors' clinical experience, it is estimated that around $2 \%-15 \%$ of patients take an authorized treatment break. The MS Choices survey found that, overall, $\sim 17 \%$ of patients were thought to have taken a treatment break. That the authors' personal experience is consistent with the findings of this survey of a wider group of physicians suggests that a significant proportion of patients (up to $\sim 20 \%$ ) are probably taking breaks in their treatment following a discussion of the pros and cons with their physician. Authorized treatment breaks may have benefits for many patients. A flexible approach to treatment, such that patients are permitted to take a short break (eg, during vacations), could increase the likelihood that a patient will stay on therapy in the longer-term. Permitted flexibility may show patients that their physicians understand their desire to be free from the burden of treatment for a short period, and may thus strengthen the patient-physician relationship.
An understanding approach fosters open discussion and provides the opportunity for physicians to advise patients on how to adapt their treatment routine to provide flexibility while avoiding missed injections. Furthermore, open discussions allow physicians to explain the risks associated with even short-term interruptions, and to remind patients of the importance of continuing therapy over the long-term.

Realistically, it is likely that many, if not all, patients will at some point choose to miss some injections. In some instances, a flexible approach to treatment in the short-term may enable the patient to remain on treatment for the longterm; however, the potential benefits of treatment breaks have not been studied. In light of the lack of good-quality trial data to inform clinicians' decisions, it is difficult to advise when and in whom treatment breaks may be appropriate. Of course, ideally all patients would stay on uninterrupted treatment, but both the MS Choices survey and previous studies suggest that this is not the reality. Therefore, it is suggested that interrupting treatment should probably only be considered for patients who have had 2-3 years of stable disease, and would caution against authorizing a treatment break in patients with evidence of disease activity despite treatment. Furthermore, disease reactivation has been reported upon stopping treatment after only a short treatment period. ${ }^{21}$ However, readers should be reminded that this recommendation is based primarily on the authors' clinical experience, not trial data, and are urged to consider each individual case carefully before making a decision.

\section{Improving adherence in clinical practice}

How to improve adherence is a complex problem, and may require several approaches (Table 1). As indicated in the survey, adherence is influenced by many variables, including side effects, how well the treatment fits with the patient's lifestyle, demands of work and family, and planned pregnancy. The MS Choices survey highlighted that patients' concerns about adherence to their MS therapy differ from the issues that physicians

Table I Strategies to improve adherence to multiple sclerosis therapies: patient-physician and patient-nurse interactions

- Understanding the factors that predict adherence

- Maintaining good patient-physician or patient-nurse communication

- Educating patients on the importance of adhering to therapy

- Educating physicians/nurses regarding issues that are important to patients

- Identifying patient-specific barriers to adherence

- Managing adverse events to preserve quality of life

- Managing patient expectation of therapy and setting realistic goals

- Identifying and setting manageable treatment schedules 
consider most important. This finding again suggests there is a need for physician education regarding the issues that are important to patients. The medical team must learn to understand what is important to patients, and to talk in terms that are meaningful if they hope to influence adherence positively.

\section{Motivating patients to continue treatment}

Maintaining adherence over the long-term can be problematic. Keeping patients motivated requires a long-term commitment from the healthcare team - patients who have been on treatment for many years should not be overlooked. Various strategies may help to achieve good long-term adherence and should be considered (Table 2). Education and maintaining motivation need to be regular events; indeed, frequent patient-physician/nurse interaction is key to a successful outcome. Physicians should ideally be trained in techniques to communicate better with and motivate patients, recognizing that these techniques will need to be tailored to the individual. This might be achieved through residential courses dedicated to professionals. A pilot study, based on experience with the "Sapere Migliora (knowing improves)" information aid, has been performed in Italy with the financial support of the Italian MS Society, and demonstrated the benefits to patients of providing additional information on their disease in an accessible format. ${ }^{22,23}$

Table 2 Barriers to long-term adherence and potential solutions

\begin{tabular}{|c|c|}
\hline Barrier & Potential solutions \\
\hline Treatment fatigue & $\begin{array}{l}\text { Educate patients on the implications } \\
\text { of taking treatment breaks or stopping } \\
\text { therapy } \\
\text { Discussion forums to remotivate } \\
\text { patients } \\
\text { Reeducate patients regarding treatment } \\
\text { expectations } \\
\text { Revisit lifestyle issues }\end{array}$ \\
\hline $\begin{array}{l}\text { Injection-site reactions/ } \\
\text { local side effects }\end{array}$ & $\begin{array}{l}\text { Nurse education on side-effect } \\
\text { management } \\
\text { Guidelines for the management of } \\
\text { injection-site reactions } \\
\text { Reinforcement of correct injection } \\
\text { technique/need to rotate injection sites } \\
\text { to avoid injection-site reactions }\end{array}$ \\
\hline Depression & $\begin{array}{l}\text { Screening for depressive symptoms } \\
\text { Treatment of depressive symptoms }\end{array}$ \\
\hline $\begin{array}{l}\text { Multiple sclerosis-related } \\
\text { cognitive decline }\end{array}$ & $\begin{array}{l}\text { Support mechanisms } \\
\text { Regular education forums } \\
\text { Reminders of treatment timing (eg, } \\
\text { SMS alerts) } \\
\text { Involvement of family/carer in } \\
\text { treatment discussions }\end{array}$ \\
\hline
\end{tabular}

Abbreviation: SMS, short message service, ie, text message.
Recording patients' positive and negative views on longand short-term therapy at treatment initiation and planned follow-up visits allows patients' opinions to be reviewed periodically and can help to start a discussion on how these feelings change over time. The use of electronic patient records capturing such information for future use might enhance this practice. For example, it may be possible to include a specific field for a patient's comments at routine visits, possibly recorded using electronic tablets and standardized questionnaires. Indeed, in Germany, an electronic patient management system, in which data are captured from many sources, has been implemented successfully and is currently being evaluated further. ${ }^{24}$ In addition, access to support networks (eg, the internet and call centers) can help to resolve queries and address concerns between clinic appointments. The medical team should provide consistent communication on the nature and purpose of DMD therapy, to prevent the patient from "losing faith" in the team.

\section{Treatment reevaluation}

For long-term therapies, it is important that treatment be evaluated regularly. The authors believe this should be done at least annually and the strategy revised depending on the patient's status. Patients should be involved in treatment decisions and empowered to select treatment, following discussion with their physician. Further discussion may be required to help patients understand issues such as the need for a secondline therapy for aggressive disease. Different approaches may be required at different stages of the disease.

\section{Patient-physician relationships and decision making Patient-physician partnership}

A good relationship between the patient and the healthcare team has the potential to improve adherence, and thus treatment outcomes. The observation that adherence rates tend to be higher in clinical trials than in clinical practice ${ }^{12}$ suggests that frequent contact with the physician, nurse, or other healthcare professional can help to achieve good adherence. However, the psychological attitude of patients participating in clinical trials might also differ from that of patients being managed in routine care: the perception that they are receiving a "new" and therefore "better" treatment can have a potent placebo effect, which may be lost when the drug becomes "standard treatment." The authors do not consider it possible to suggest an optimal frequency for patient-physician contact, as the frequency of visits should be tailored to the individual. 
Alternative communication methods, such as email, between patients and physicians are becoming increasingly popular. While increased patient-physician dialog is a positive step, electronic communications may be time consuming, and physicians' responses may not achieve the same depth or completeness as a face-to-face consultation. In addition, there is also the potential for misinterpretation in such communications. In the authors' experience, patients often ask the same question several times, indicating that they may not fully understand the information that had been provided. Although over half of patients completing the MS Choices survey reported discussing aspects of their treatment other than side effects with their physician or nurse, the survey did not explore whether patients understood the information that they had been given. Clearly, to achieve a real benefit for each patient, strategies to improve patient-physician communication should be explored. Such strategies include asking patients how much they understood of what they had been told, the provision of information in different formats (eg, in direct discussions, leaflets, videos, internet), and ensuring simple terminology is used to avoid confusing or "intimidating" the patient.

Physicians and nurses should work hard to establish and maintain a good relationship with their patients. Ideally, patients should feel that they are a partner in decisions regarding the management of their disease and are able to raise concerns and talk openly with their physician/nurse. On the other hand, it is also important that patients trust their physician to make decisions that are in their best interests. In the authors' experience, patients are not always honest with their physicians regarding adherence to therapy: developing good relationships and trust between patients and physicians is essential so that patients feel that they can talk openly and honestly about their care, without fear of consequences.

\section{Involvement of patients in the treatment decision-making process}

Most physicians believed that patients should be involved in choosing their treatment, but only around a quarter of patients felt that they had discussed treatment options with their medical team and had been involved in the final treatment decision. Around half of patients followed their physician's/nurse's recommendation after discussing their treatment options with them. Interestingly, most patients were aware of the possible risks and benefits of their current MS treatment, and increasingly patients obtain information from other sources such as patient forums and the internet. In some cases, patients may ask for more information once they have started treatment so that they feel informed about, but are not involved in, treatment decisions. For young, newly diagnosed patients, structured group discussions to promote knowledge and self-management have been shown to have benefits. ${ }^{25}$

Considering the discrepancies that were seen between patient and physician responses in the MS Choices survey, it is clearly important that patients are more aware of issues surrounding their treatment, irrespective of whether they want the physician alone to make decisions regarding their treatment. The extent of patient involvement in the decision-making process is likely to be heavily influenced by cultural differences, which could explain the regional differences seen. The proportion of patients who felt fully involved ranged from $20 \%$ in Spain to $75 \%$ in Canada. These differences highlighted in the MS Choices survey largely reflect the authors' clinical experience (Table 3 ) and previous findings. ${ }^{26,27}$ Engaging the patient in treatment decisions can be empowering and has the potential to improve adherence.

\section{Role of the MS nurse in improving adherence}

The MS Choices survey did not question MS nurses or explore the relationship between patients and their MS nurse. However, the authors believe that MS nurses play a vital role in the care of patients with MS and, through their interactions with patients, can help patients adhere to their treatment. It would be interesting to know whether nurses' responses to the questions posed in the MS Choices survey would be more closely aligned with patient response than were those of physicians. In the authors experience, many patients are more comfortable discussing certain aspects of their disease and treatment, such as sexual and sphincter disturbances or side effects, with their MS nurses than with their physicians, preferring instead to talk to their physicians about treatment choices. This observation suggests that patients feel able to ask questions about their disease and treatment-related side effects with their MS nurses that they may not want to discuss with their physicians.

Discussions with a nurse tend to be more relaxed than those with a physician, and may avoid complicated medical terms that may intimidate or confuse the patient. Nurses may be more likely to translate medical terminology into terms that patients can understand easily, such as talking about how far a patient can walk, rather than using the term "Expanded Disability Status Scale score.” In some countries, nurses visit patients at home at the start of treatment. This provides 
Table 3 Opinions on regional differences in patient's attitudes to their involvement in the treatment decision-making process

\begin{tabular}{ll}
\hline Country & General patient attitude \\
\hline Spain & Do not want to choose their treatment \\
& Prefer the physician to make the decision \\
Germany & Want to be included in discussions \\
& Many wish to decide for themselves \\
United Kingdom & Willing to receive a large amount of information \\
& Want to be included in the discussions regarding choice, but often request direction \\
& and guidance from healthcare team to support them in making a decision; it differs \\
& between individuals \\
Italy & Differs between patients with different psychological profiles, cultural attitudes, \\
& and socioeconomic status
\end{tabular}

a more relaxed forum for discussion and aims to reduce anxiety that patients may feel from being in a clinical setting. That patients may view nurses differently from physicians can be exploited to the patient's advantage. Regular nurseled reviews (eg, every 6 months) can provide reassurance, advice on managing side effects, and allow open discussion to reevaluate patient expectations and address concerns. However, the extent to which MS nurses are involved in routine patient care differs between countries; for example, patients in the United Kingdom have more contact with MS nurses than with physicians. A well-developed network of neurology nurses, regular nurse training, and workshops on key issues such as side effects and adherence are important to ensure that patients receive consistent information and standard of care. That patients have different relationships with different healthcare professionals highlights the importance of ensuring that patients have regular access to both nurses and physicians.

\section{Psychological patient profiling}

The MS Choices survey highlighted numerous differences both between patients and physicians and between patients from different regions. It is important to remember to treat patients as individuals: psychological profiling is a potentially powerful but little used tool that can help physicians to understand the individual patient's wants and needs. Psychological patient profiling, although not widely used, allows the "categorization" of patients based on considerations such as emotional state. Profiling can help to identify who should be started earlier or later on treatment, and can help to decide on the best approach to gain patients' acceptance of their situation and to initiate discussions on treatment options. Profiling can also help to determine the extent to which a patient will want to be involved in treatment decisions and to predict who is more or less likely to adhere to treatment and an individual's reasons for nonadherence.
To achieve the greatest benefit, profiling should involve a clinical psychologist and a neuropsychologist as part of a multidisciplinary team. Assessments should be conducted at specific milestones. At the minimum, these should be when informing the patient of the diagnosis (both before and after the diagnosis is given), when starting treatment (both before and after the decision on treatment has been made), and when problems arise (eg, following a relapse or when a patient wants to discontinue treatment).

\section{Recommendations based on lessons from MS Choices}

Based on the findings of the MS Choices survey, and personal clinical experience in various countries across Europe, the authors would like to propose some recommendations regarding how to maximize adherence in clinical practice (Table 4). Crucially, strategies should be determined on an individualpatient basis and after engaging the patient in decisions regarding their treatment, as appropriate for the individual.

Table 4 Recommendations for maximizing patient adherence in clinical practice

\footnotetext{
- Psychological profiling should be performed to determine patients':

- Attitude to their disease

- Attitude to treatment and motivating factors

- Desire to be actively involved in treatment decisions

- Positive and negative perceptions of long- and short-term treatment

- Preferred communication methods

- Physicians should be educated regarding:

- Issues that are important to patients

- Factors that form barriers to adherence

- The need to regularly reinforce the importance of good adherence

- Patients need educating regarding the importance of good adherence

- Regular and frequent discussions should take place to:

- Ensure patients' concerns are addressed

- Continually motivate patients to stay on treatment

- An interdisciplinary team approach should be employed

- Patients should have regular access to their multiple sclerosis nurse
} 


\section{Conclusion}

The MS Choices survey revealed differences between patients and physicians concerning several aspects of treatment, notably in the factors leading to treatment breaks or discontinuations. Regional differences between countries were also identified, and which may reflect cultural and/or economic influences, which should be given due consideration during patient consultations. The findings of the survey confirmed that treatment adherence is an important and complex issue in MS; here, barriers to adherence and potential approaches to improving adherence were discussed. For patients requiring chronic treatment, possibly over several decades, it is probably not realistic to expect them to achieve "total" adherence. A flexible, individualized approach may be more appropriate, to include authorized treatment breaks after careful consideration of potential risks. Psychological profiling can help to identify factors that are important to, and that will motivate, individual patients. A good relationship between patients and their medical team is essential. Patients should be involved in treatment decision making, should they so wish, and should have realistic expectations of therapy. Regular discussions should take place between patients and their physician or nurse, preferably both.

\section{Acknowledgments}

The authors thank Andrea Plant and Joanne Tang of Caudex Medical Ltd, Oxford, United Kingdom (supported by Merck Serono S.A. - Geneva, Switzerland, a branch of Merck Serono S.A. Coinsins, Switzerland, an affiliate of Merck KGaA, Darmstadt, Germany) for assistance with the preparation of the initial draft of the manuscript and for collating input from all authors (AP), and for English editing and assistance with preparation for submission (JT).

\section{Disclosure}

Alessandra Lugaresi has been an advisory board member for Biogen Idec, Merck Serono, and Bayer Schering; has received travel grants and honoraria from Bayer Schering, Biogen Dompé, Merck Serono, Novartis, Sanofi-Aventis, and Teva; has received research grants from Bayer Schering, Biogen Dompé, Merck Serono, Novartis, and Sanofi-Aventis; has received travel and research grants from the Associazione Italiana Sclerosi Multipla; and is a consultant for "Fondazione Cesare Serono."

Tjalf Ziemssen has received speaker honoraria from Biogen Idec, Sanofi-Aventis, Merck Serono, MSD, Novartis, Synthon, Teva, and Bayer HealthCare; and serves as a consultant for Merck Serono, Roche, Synthon, Teva, Novartis, and Bayer HealthCare.

Celia Oreja-Guevara has received honoraria as consultant on scientific advisory boards by Bayer Schering, Merck Serono, Biogen, Teva, and Novartis, and has participated in clinical trials and other research projects supported by Biogen Idec, GSK, Teva, and Novartis.

Delyth Thomas has received honoraria and consultation fees from Merck Serono and Novartis.

Elisabetta Verdun is an employee of Merck Serono S.A. - Geneva, Switzerland, a branch of Merck Serono S.A. Coinsins, Switzerland, an affiliate of Merck KGaA, Darmstadt, Germany.

\section{References}

1. World Health Organization. Adherence to long-term therapies: evidence for action. August 11, 2003. Available from: http://www.who.int/chp/ knowledge/publications/adherence_report/en/index.html. Accessed December 1, 2009.

2. Treadaway K, Cutter G, Salter A, et al. Factors that influence adherence with disease-modifying therapy in MS. J Neurol. 2009;256(4): 568-576.

3. Halpern R, Agarwal S, Dembek C, Borton L, Lopez-Bresnahan M. Comparison of adherence and persistence among multiple sclerosis patients treated with disease-modifying therapies: a retrospective administrative claims analysis. Patient Prefer Adherence. 2011;5: 73-84.

4. Tremlett HL, Oger J. Interrupted therapy: stopping and switching of the beta-interferons prescribed for MS. Neurology. 2003;61(4):551-554.

5. Tremlett H, Van der Mei I, Pittas F, et al. Adherence to the immunomodulatory drugs for multiple sclerosis: contrasting factors affect stopping drug and missing doses. Pharmacoepidemiol Drug Saf. 2008;17(6):565-576.

6. Rio J, Porcel J, Tellez N, et al. Factors related with treatment adherence to interferon beta and glatiramer acetate therapy in multiple sclerosis Mult Scler. 2005;11(3):306-309.

7. Portaccio E, Zipoli V, Siracusa G, Sorbi S, Amato MP. Long-term adherence to interferon beta therapy in relapsing-remitting multiple sclerosis. Eur Neurol. 2008;59(3-4):131-135.

8. Cox D, Stone J. Managing self-injection difficulties in patients with relapsing-remitting multiple sclerosis. J Neurosci Nurs. 2006;38(3): 167-171.

9. Tan H, Cai Q, Agarwal S, Stephenson JJ, Kamat S. Impact of adherence to disease-modifying therapies on clinical and economic outcomes among patients with multiple sclerosis. Adv Ther. 2011;28(1) 51-61

10. Al-Sabbagh A, Bennet R, Kozma C, Dickson M, Meletiche D. Medication gaps in disease-modifying drug therapy for multiple sclerosis are associated with an increased risk of relapse: findings from a national managed care database. J Neurol. 2008; 255(Suppl 2):321.

11. Haynes RB, Ackloo E, Sahota N, McDonald HP, Yao X. Interventions for enhancing medication adherence. Cochrane Database Syst Rev. 2008;(2):CD000011.

12. Lugaresi A. Addressing the need for increased adherence to multiple sclerosis therapy: can delivery technology enhance patient motivation? Expert Opin Drug Deliv. 2009;6(9):995-1002.

13. Pozzilli C, Schweikert B, Ecari U, Oentrich W. Supportive strategies to improve adherence to IFN beta-1b in multiple sclerosis - results of the BetaPlus observational cohort study. J Neurol Sci. 2011;307(1-2): $120-126$. 
14. Mikol D, Lopez-Bresnahan M, Taraskiewicz S, Chang P, Rangnow J. A randomized, multicentre, open-label, parallel-group trial of the tolerability of interferon beta-1a (Rebif $\left.{ }^{\sqrt{ }}\right)$ administered by autoinjection or manual injection in relapsing-remitting multiple sclerosis. Mult Scler. 2005;11(5):585-591.

15. Brochet B, Lemaire G, Beddiaf A. Reduction of injection site reactions in multiple sclerosis (MS) patients newly started on interferon beta $1 \mathrm{~b}$ therapy with two different devices. Rev Neurol (Paris). 2006;162(6-7): 735-740. French.

16. Riñón A, Buch M, Holley D, et al. The MS Choices Survey: findings of a study assessing physician and patient perspectives on living with and managing MS. Patient Pref Adherence. 2011;5:629-643.

17. Devonshire V, Lapierre Y, MacDonell R, et al. The Global Adherence Project - a multicentre observational study on adherence to disease-modifying therapies in patients suffering from relapsing-remitting multiple sclerosis. Mult Scler. 2006;12 (Suppl 1):S229-S243.

18. Devonshire V, Lapierre Y, Macdonell R, et al. The Global Adherence Project (GAP): a multicenter observational study on adherence to disease-modifying therapies in patients with relapsing-remitting multiple sclerosis. Eur J Neurol. 2011;18(1):69-77.

19. Heesen C, Kleiter I, Nguyen F, et al. Risk perception in natalizumab-treated multiple sclerosis patients and their neurologists. Mult Scler. 2010;16(12):1507-1512.

20. Kern S, Schrempf W, Schneider H, Schultheiss T, Reichmann H, Ziemssen T. Neurological disability, psychological distress, and healthrelated quality of life in MS patients within the first three years after diagnosis. Mult Scler. 2009;15(6):752-758.
21. Durelli L, Bongioanni MR, Ferrero B, et al. Interferon alpha-2a treatment of relapsing-remitting multiple sclerosis: disease activity resumes after stopping treatment. Neurology. 1996;47(1):123-129.

22. Borreani C, Giordano A, Falautano M, et al. Experience of an information aid for newly diagnosed multiple sclerosis patients: a qualitative study on the SIMS-Trial. Health Expect. 2011. [Epub ahead of print.] doi: 10.1111/j.1369-7625.2011.00736.x.

23. Solari A, Martinelli V, Trojano M, et al. An information aid for newly diagnosed multiple sclerosis patients improves disease knowledge and satisfaction with care. Mult Scler. 2010;16(11):1393-1405.

24. Schultheiss T, Kempcke R, Kratzsch F, et al. [Multiple sclerosis management system 3D: moving from documentation towards management of patients]. Nervenarzt. 2011. [Epub ahead of print.] doi: 10.1007/s00115-011-3376-6. Article in German.

25. Kern S, Reichmann H, Ziemssen T. Adherence to neurologic treatment. Lessons from multiple sclerosis. Nervenarzt. 2008;79(8):877-886. German.

26. Heesen C, Kasper J, Segal J, Kopke S, Muhlhauser I. Decisional role preferences, risk knowledge and information interests in patients with multiple sclerosis. Mult Scler. 2004;10(6):643-650.

27. Giordano A, Mattarozzi K, Pucci E, et al. Participation in medical decision-making: attitudes of Italians with multiple sclerosis. J Neurol Sci. 2008;275(1-2):86-91.
Patient Preference and Adherence

\section{Publish your work in this journal}

Patient Preference and Adherence is an international, peer-reviewed, open access journal focusing on the growing importance of patient preference and adherence throughout the therapeutic continuum. Patient satisfaction, acceptability, quality of life, compliance, persistence and their role in developing new therapeutic modalities and compounds to

\section{Dovepress}

optimize clinical outcomes for existing disease states are major areas of interest. This journal has been accepted for indexing on PubMed Central. The manuscript management system is completely online and includes a very quick and fair peer-review system. Visit http://www.dovepress.com/ testimonials.php to read real quotes from published authors. 